\title{
Die Perzeption als Arbeitsgebiet in der Humangeographie
}

\section{Geographie und Perzeption}

Seit den späten sechziger Jahren zeichnet sich immer mehr eine verstärkte Orientierung der Humangeographie auf den Menschen ab. Sie findet ihren Ausdruck einerseits im Bezug auf das räumliche Verhalten des Menschen (Hartke 1959, Boustedt 1964, Wolpert 1965, Cox \& Golledge 1969, Doherty 1969, Cox 1972, Thomale 1974 u.a.m.), andererseits im Interesse an der Umweltwahrnehmung (Gould 1965, Gould \& White 1968, 1974, Lowenthal 1967, Brookfield 1969, Saarinen 1969, Downs 1970, Goodey 1973, Corna Pellegrini 1979 u.a.m.). Gould formulierte schon 1965 als wesentliches Ziel der Ausweitung der Humangeographie als "emphasizing the human as much as the geography" (Gould 1965, 182, Hervorhebung im Origina1). Erk1ärungen für das menschliche Verhalten im Raum und die daraus resultierenden Prozesse und Strukturen werden sowohl von ihm aus als auch vom Raum her gesucht.

Der Mensch ist Akteur im und Aktivator des Raumes, seine Entscheidungen sind durch zahlreiche, z.T. rationale, z.T. irrationale Faktoren bestimmt. Zu den Irrationalen zäh1t der subjektive Raum oder das Image. Dieses ist an sich individuell, von einem Menschen zum andern verschieden. Der Subjektivierungsvorgang wird durch mehrere Einflüsse gesteuert (Fig. 1). Zweifellos spielt dabei die Information eine wesentliche Rolle, doch sind auch die beiden andern Faktoren nicht zu vernachlässigen: Wertesysteme sind kulturell bedingt und spiegeln die gängigen Grundhaltungen wider, während die psychologische Grundhaltung die Einstellung des Einzelnen darstellt. Da diese 3 Faktoren nie fest sind, ist auch das Image ständigen Wandlungen unterworfen. 1)

Unter solchen Voraussetzungen kann die Humangeographie nicht mehr nur strukturell orientiert sein, sondern muss sich auf räumliche Prozesse beziehen. Sie orientiert sich am subjektiv bedingten räumlichen Verhalten des Menschen und muss dabei auch die irrationalen Komponenten der Entscheide berücksichtigen. Damit ist eine gewisse Hinwendung zur Psychologie verbunden. Wahrnehmungs- (Hajos 1972) und Umweltpsychologie (Mercer 1975, Canter 1977) sind in unterschiedlichem Masse Hilfswissenschaften für diesen neuen Ansatz.

Der reale Raum wird vom Menschen subjektiv erlebt, und sein räunliches Bild stützt sich auf diese Erfahrung ab. Sie ist im wesentlichen eine Erfahrung des täglichen Lebens. Das subjektive Bild der Umwelt ist das Resultat des Wahrnehmungsprozesses, der in der Literatur auch als Perzeption oder Umweltwahrnehmung bezeichnet wird. In der Tat ist die Verwendung dieser Begriffe etwas unscharf, indem sie sowohl den Wahrnehmungsprozess als auch das Resultat der Wahrnehmung (das Wahrgenommene oder das Erkannte) damit bezeichnen. 2) Perzeption oder Wahrnehmung kann als Filter gesehen werden: gewisse Teile der objektiven Welt bleiben darin hängen, werden vom Menschen nicht aufgenommen, andere werden bewusst, weitere unbewusst erfasst und im Gehirn gespeichert (Fig. 2). Rezeptoren sind die Sinnessysteme, die uns erlauben, den Raum zu erkennen und wahrzunehmen. Am bedeutendsten sind dabei wohl die Augen, doch spielen auch das Gehör, der Geruchund der Tastsinn sowie das Empfindungsvermögen für Temperaturen eine Rolle. Wir können z.B. eine Geräuschquelle auch dann lokalisieren, wenn wir sie nicht sehen. Die Rolle der einzelnen Sinnessysteme ist unterschiedlich und variiert je nach kulturellen Gegebenheiten und Grad der Vertrautheit mit der Natur (vgl. Tuan 1974, pp. $6 \mathrm{ff}$, $13 \mathrm{ff}, 59 \mathrm{ff}, 75 \mathrm{ff}$ ).

Dr. Walter Leimgruber, Geographisches Institut der Universität Basel, Klingelbergstr. 16, 4056 Basel

1) Lynch bemerkt dazu: "Environmental images are the results of a two-way process between the observer and his environment. The environment suggests distinctions and relations, and the observ er - with great adaptability and in the light of his own purposes - selects, organizes, and endows with meaning what he sees. The image so developed now limits and emphasizes what is seen, while the image itself is being tested against the filtered perceptual input in a constant interacting process. Thus the image of a given reality may vary significantly between different observers." (Lynch 1960: 6). 


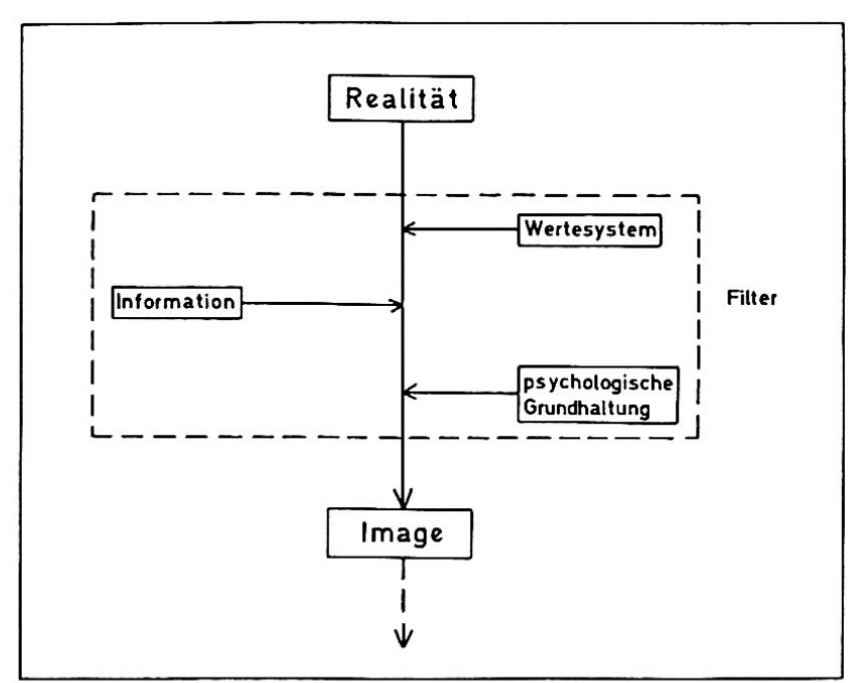

Fig.1: Konzeption der Imagebildung (nach J.Doherty, verändert).

Welche Rolle kommt dieser Forschungsrichtung in der Geographie überhaupt zu? Soll sie wirklich auf die Psychologie zurückgreifen, oder begeben sich hier die Geographen auf ein Nebengleis, das leicht zu einem Abstellgleis werden könnte?

Wir befinden uns ungefähr in der gleichen Lage wie zur Zeit, als die Geographie erste Ergebnisse der Soziologie zu übernehmen begann. Damals befürchtete man, die Humangeographie würde von der Soziologie überspielt. Inzwischen sind die Dinge ins rechte Licht gerückt worden, und man hat gelernt, Erkenntnisse und Arbeitsmethoden aus diesem Fachbereich in der Geographie anzuwenden. Eine Abwehr- oder gar Isolationsstellung ist darum fehl am Platz, weil es nicht darum geht, die Humangeographie zu psychologisieren, sondern
Ergebnisse und Methoden der Psychologie anzuwenden, die in der Geographie neue Erkenntnisse bringen.

\section{Anwendungsbereiche}

Ein Blick auf die schon ins Unüberschaubare angewachsene Literatur zeigt, dass die Geographie von der Psychologie über diePerzeptionsforschung wertvolle Anregungen erhalten kann. Ich sehe dabei drei verschiedene Gebiete:

1. Die didaktische Anwendung in der Schule. Die Anwendung von Arbeitsmethoden der Umweltperzeption kann zu einer bewussten Auseinandersetzung der Schüler mit dem Raum führen und das räumliche Abstraktionsvermögen entwickeln helfen. Wie S. Catling (1979) ausführte, besitzen Kinder bereits im Primarschulalter ein beachtliches räumliches Vorstellungsvermögen, das ausbaufähig ist. Ergebnisse der Perzeptionsforschung können dabei wertvolle Dienste leisten (vgl. auch Geipel 1974).

2. Die vielzitierte Beteiligung der Oeffentlichkeit am Planungsprozess wird zur Farce, wenn das Publikum keine räumliche Vorstellung über Planungsraum hat. Können der Bürger, der Parlamentarier und der Politiker überhaupt einen Plan lesen und interpretieren, über den sie entscheiden müssen, können sie seine räumliche Tragweite ermessen? Wie sieht der Bürger den zu beplanenden Raum? Was für Vorstellungen hat er vom aktuellen Zustand, von den ablaufenden raumwirksamen Prozessen, vom wünschbaren $\mathrm{Zu}-$ stand? Eine aktive und fruchbare Beteiligung kann nur erreicht werden, wenn der Einzelne sich um solche Fragen kümmert, und wenn die Planer bereit sind, an das Publikum heranzutreten, um seine Meinung zu erfahren. Leser-

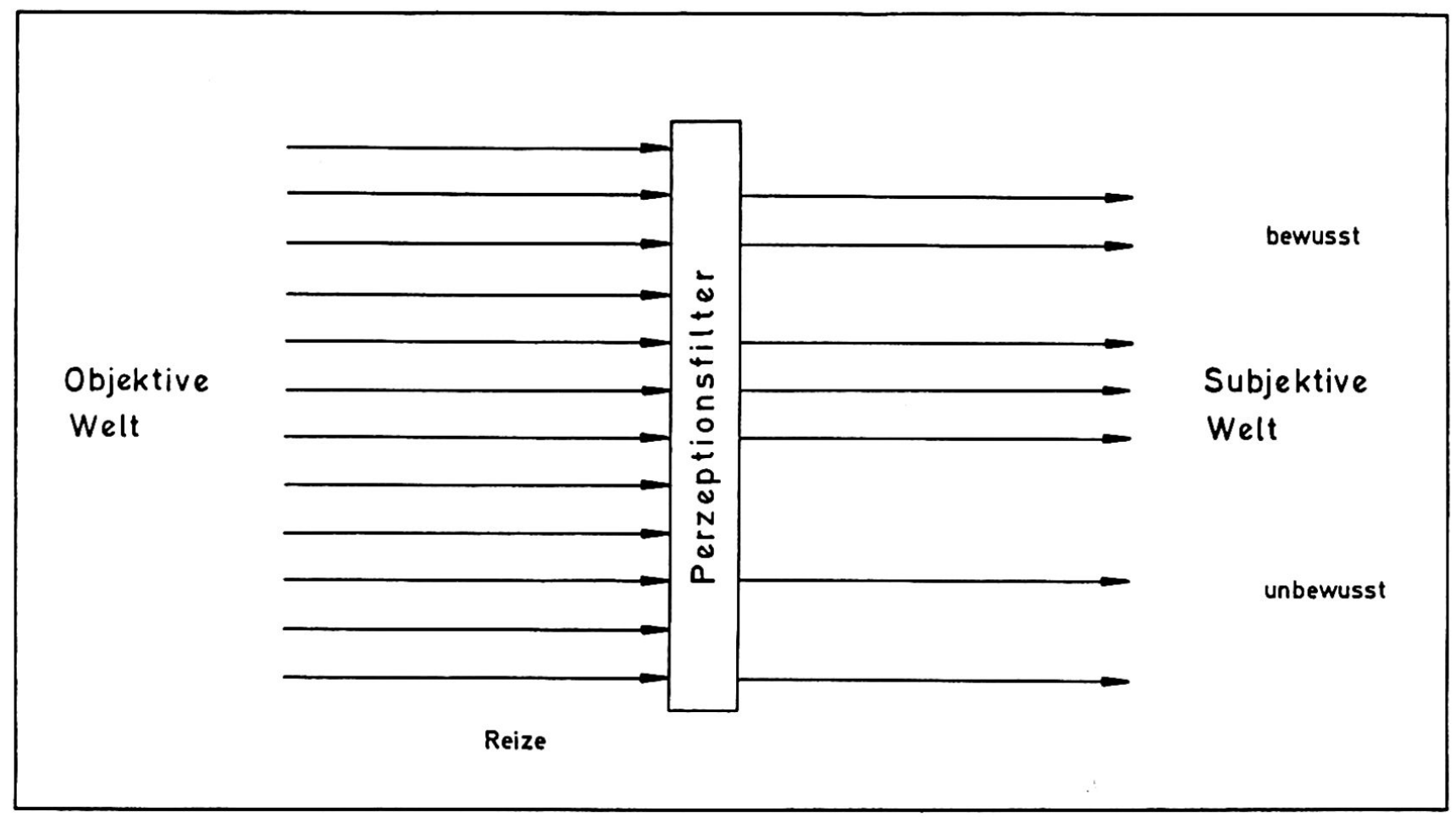

Fig.2: Die Umweltwahrnehmung als Filter. 
briefe allein sind keine Stellungnahmen der Oeffentlichkeit. Das gilt für Fragen der Ortsbzw. Stadt- und Regionalplanung genauso wie für den Umweltschutz und die Vorsorge vor $\mathrm{Na}-$ turgefahren (hazard research).

3. In der humangeographischen Forschung ist die Unweltwahrnehmung ein Arbeitsinstrument einer verhaltensorientierten Geographie. Der Mensch fällt seine räumlichen Entscheide und agiert in Raum u.a. aufgrund seiner 'mental map" (Raumvorstellung; zum Begriff vgl. Kishimoto 1975). Sie ist eine der irrationalen Komponenten, die wir zur Erklärung des räumlichen Verhaltens verwenden können. Dabei geht es nicht darum, kausale Verknüpfungen zu suchen, sondern die Frage lautet: Warum hat der Mensch gerade diese Handlungsweise gewählt und nicht eine alternative, die vorhanden wäre (Berry 1973, 13)?

Dieser Ueberblick zeigt, dass der Umweltperzeption sowohl im Hinblick auf die humangeographische Forschung als auch auf die Praxis in Zukunft verstärkte Bedeutung zukommen wird. Dabei muss aber mit Nachdruck darauf hingewiesen werden, dass es nicht genügt, einfach serienweise "mental maps" ausfindig zu machen. Wichtig sind'mental maps" (z.B. bezogen auf soziale Gruppen oder spezielle regionale Bedingungen) im Rahmen der Erklärungen und des Verstehens räumlichen Verhaltens.

\section{Forschungsrichtungen}

Die Studien, die unter dem Titel "Perzeption" laufen, lassen sich sowohl der Aktual- wie auch der historischen Geographie zuordnen (zum letzteren vgl. einzelne Aufsätze in Corna Pellegrini 1979, dazu auch Brusa 1979). Goodey (1973) zählt insgesamt 9 Bereiche von Perzeptionsstudien auf: Unweltqualität, Gefahren, Bild der Stadt, Perzeption von Routen her, Grenzen, persönlicher Raum, entfernte Gebiete, Präferenzräume, Planungsmitarbeit und Erziehung. Eine genauere Betrachtung zeigt, dass mehrere dieser Bereiche sich nur im Massstab voneinander unterscheiden (z.B. Stadt und entfernte Gebiete, Umweltqualität und -gefahren). Mir scheint, dass sich eigentlich die drei folgenden Hauptbereiche unterscheiden lassen:

1. Kenntnis des Raumes. Untersuchungen zu diesem Thema behandeln in der Regel entweder den 10kalen Rahmen (die Stadt) oder aber ein Land, einen Kontinenten oder die ganze Erde. Am bekanntesten sind zweifellos Arbeiten zum Bild der Stadt, beginnend mit dem Klassiker von Kevin Lynch (1960, 14. Nachdruck 1977). Goodey (1973, $23 \mathrm{ff})$ führt eine ganze Anzahl Studien auf. Bemerkenswert ist die Untersuchung von Peter Orleans (1973) über das Bild von Los Angeles bei verschiedenen sozialen Gruppen unter den Bewohnern. Von den Arbeiten über weltweite oder nationale Räume sei diejenige von Saarinen (1973) erwähnt. Sie zeigt, wie die befragten Studenten die einzelnen Kontinente und Länder strukturieren. Faktoren wie
Ethnozentrizität, Nachbarschaft, Präferenzen (Wunschträume) etc. bestimmen das Bild. Seine Schlussfolgerungen sind weitreichend: Die Angehörigen der einzelnen Länder, u.U. einzelner Nationengruppen, haben verschiedene räumliche Vorstellungen von der Welt; Untersuchungen dieser Art (mittels freihändig gezeichneter Kartenskizzen) können helfen, Mängel in der geographischen Erziehung aufzuzeigen und damit zu einem besseren Verständnis unter den Menschen führen. Sicher ist es aber nicht allein die "mental map" der Schüler und Studenten, die hierzu dienen kann, es ist vielmehr auch eine Erziehung zum Verständnis anderer Kulturen und Wertsysteme.

2. Raumpräferenzen. Der Begriff "mental map" wurde meines Wissens erstmals 1965 von Peter Gould (1965/1973) verwendet, als Synonym zu "spatial images" (räumliche Bilder bzw. Karten im menschlichen Geist). Gould basierte seine Untersuchungen auf der Vorliebe der Befragten für bestimmte Räume. Die Testpersonen mussten angeben, wo sie sich bei vollkommen freier Wahl am liebsten niederlassen wollten. Befragt wurden vor allem Studenten und Schüler, d.h. eine junge, zur Mobilität neigende Bevölkerungsgruppe. Mit dieser Methode hat er wesentlich zur Verbreitung der Umweltwahrnehmung als geographisches Forschungsgebiet beigetragen (vg1. auch Gould 1975, Gould \& White 1968, 1974).

Raumpräferenzen sind ein wichtiges Element beim Studium von Wanderungsbewegungen. Somit eignen sie sich vor allem für Untersuchungen auf nationaler oder internationaler Ebene. Auf regionaler Ebene jedoch scheint dieses Verfahren nicht sehr aussichtsreich zu sein, da hier zu viele Zufälligkeiten mitspielen. Zudem dürften Raumpräferenzen auch in nationalem Massstab nur als ein Erklärungselement gelten, da stereotype Vorstellungen wie "romantisch", "schön" etc. auf die Wahl der bevorzugten Orte einwirken. Wird die Frage nicht nach dem Wohn- sondern nach dem Arbeitsort oder sogar nach der bevorzugten Erholungsregion gestellt, so dürfte sich die "mental map" der gleichen Bevölkerungsgruppe erheblich ändern. Was also aufgrund einer einzigen Frage als die 'mental map" herausgestellt wird, entpuppt sich bei näherem Betrachten als eine "mental map" von mehreren, die wir mit uns herumtragen.

3. Raumbewertung. Schon Gould hat erkannt, dass die Präferenzräume allein nicht genügen. Bei einer Untersuchung in Tanzania und Ghana hat er deshalb auch noch die Raumbewertung berücksichtigt (Gould \& White 1974, $160 \mathrm{ff}$ ). Die einzelnen Regionen mussten mittels eines semantischen Profils zu den 5 Themen Lohn - Zugänglichkeit - Umgebung - Freizeitaktivitäten - Bevölkerung bewertet werden. Das soziologische Arbeitsinstrument des Polaritätsprofils bewährt sich in diesem Zusanmenhang, denn die räumlichen Entscheidungen beruhen auch auf solchen Bewertungen, nicht nur auf dem räumlichen Bild, das wir uns von einer Region machen. 
Mit zur Raumbewertung können wir auch die "hazard perception" rechnen: wie gross beurteilt der Bewohner einer Gegend die Gefahr von Ueberschwemmungen, Dürre, Stürmen, Erdbeben, Lawinen, Erdrutschen etc.? Dass häufig die Gefahren unterschätzt oder ignoriert werden, ist eine überraschende Feststellung. Entweder steckt eine fatalistische Grundhaltung dahinter (man will nichts davon wissen), oder aber seit dem letzten Unglück ist soviel Zeit verstrichen, dass es aus dem Bewusstsein verschwunden ist. Dass neben natürlichen auch menschliche Gefahren mit zur Raumbewertung herangezogen werden können, zeigt die Arbeit von David Ley (1972, zit. in Gould \& White 1974 , p. $30 \mathrm{f}$ ).

Perzeption als humangeographisches Arbeitsinstrument

Wie bereits gesagt, dient die Unweltwahrnehmung dazu, das menschliche Verhalten im Raum verstehen zu helfen, ein Verhalten, das zu unterschiedlichen Teilen auf rationalen und irrationalen Faktoren beruht. Eigentliche Arbeiten dazu sind bis jetzt nur wenige entstanden. Ruhl (1971) hat in seiner Diplomarbeit einen Perzeptionsansatz verwendet, und er darf damit als ein Pionier im deutschsprachigen Raum angesehen werden. $\mathrm{Zu}$ seiner Zeit waren die ersten Arbeiten von Peter Gould bekannt geworden (Gould 1965, Gould \& White 1968), sowie die theoretischen Grundlagen von Wolpert (1965). Diese Autoren liefern denn auch die Basis für Ruh1s Untersuchung. Er verwendet einen Präferenzansatz, um die Zuwanderung nach München zu erklären. Während aber Gould \& White eher die potentielle Wanderungsbewegung zu ergründen suchen und sich auf die Befragung von Schulentlassenenen abstützten, ging Ruhl auch der tatsächlichen Wanderung nach und schloss Neuzuzüger in seine Umfrage ein. Er ermittelte das Vorstellungsbild oder Image von München, und zwar sowoh1 als Selbstimage (aus Zeitungsaufsätzen) wie auch als Fremdimage (über die Fragebogen) und versuchte herauszufinden, ob es sich auf den Zuwanderungsentscheid auswirkte. Seine Umfrage umfasste einerseits über 6000 Gymnasiasten aus der ganzen BRD (potentielle Zuwanderer, Zufallsstichprobe), andererseits 90 Neuzuzüger nach Minchen kurz nach ihrem Domizilwechsel. Diese letztere Gruppe erscheint zahlenmässig etwas schwach, doch ergab sich das aus der Auswahl: nur Zuzüger aus dem $N$ und NW der BRD wurden gewäh1t, weil für Leute aus Süddeutschland eine Zuwanderung ins Oberzentrum München anders motiviert ist als für einen Einwohner aus Frankfurt. Zudem wurden nur Personen in höheren und leitenden Stellungen befragt, da die Zuwanderung weniger mit beruflichen Ambitionen als mit immateriellen Motiven (kulturelles Angebot, Freizeitmöglichkeiten) erklärt werden kann. - Ruhl kommt aufgrund der Untersuchung zum Ergebnis, dass das Image von Mïnchen so positiv sei, dass die Stadt noch auf lange Sicht Zuwanderungspol bleiben wird. Es kann also nicht als lenkende Massnahme für die Einwanderung angewendet werden. Nötig wäre allenfalls, das Image der Unterzentren der
Region zu heben, damit ein Teil der Zuwanderer aus der Stadt in die Region gelenkt werden könnten. Seine Studie zeigt deutlich, dass der Präferenzansatz, wenn er entsprechend ausgebaut wird, eine wertvolle Hilfe für humangeographische Forschung sein kann, indem ein Faktum (starke positive Wanderungsbilanz, Bevölkerungsdruck in einem Stadtraum) durch einen psychologischen Faktor (das Vorstellungsbild) hinreichend erklärt werden kann. Da die Daten aggregiert sind, bleiben individuelle andere Gründe von der Betrach-. tung ausgeschlossen. Es wäre also verfehlt $\mathrm{zu}$ sagen, der Perzeptionsansatz bringe nun die Lösung aller Probleme.

Eine laufende Forschungsarbeit in der Grenzregion TI - Italien arbeitet ebenfalls mit einem Perzeptionsansatz (Leimgruber 1979 b). Die Thematik - "Regionale Disparität und räumliche Interaktion in Grenzregionen" - verlangt aber ein anderes Vorgehen. Priorität kommt dem Faktor der politischen Grenze und der Grenzregion zu, wobei weniger die nationale Grenzregion als vielmehr die grenzüberschreitende (internationale) Region interessiert. Die Perzeption tritt dabei als Hilfsmittel auf. Ausgangspunkt ist das Mode11, das Joseph Sonnenfeld 1969 entworfen hat (vgl. Saarinen 1969, Sonnenfeld 1972). Nach ihm können wir eine Reduktion der totalen objektiven Unwe1t in drei Stufen erkennen: in den Tätigkeitsraum (operational environment), in den Wahrnehmungsraum (perceptional environment) und in den Aktions- oder Verhaltensraum (behavioral environment). Unser Aktions- oder Verhaltensraum ist enger als unser Wahrnehmungsraum, aber auf diese beiden wirkt ein noch viel weiterer Raum ein, eben der "Tätigkeitsraum" (dt. Begriff von Thomale 1974), in dem sich entscheidende sozioökonomische Prozesse abspielen. Diese drei "Räume" sind also miteinander verknüpft, wirken gegenseitig aufeinander ein. Daraus ergibt sich das Zusammenspiel der drei Elemente Interaktion - Perzeption - Prozess, die jeweils einem dieser drei Räume entsprechen (Fig. 3). Das räumliche Vorstellungsbild beeinflusst den Aktionsraum, die räumliche Interaktion gibt gleichzeitig zu einer

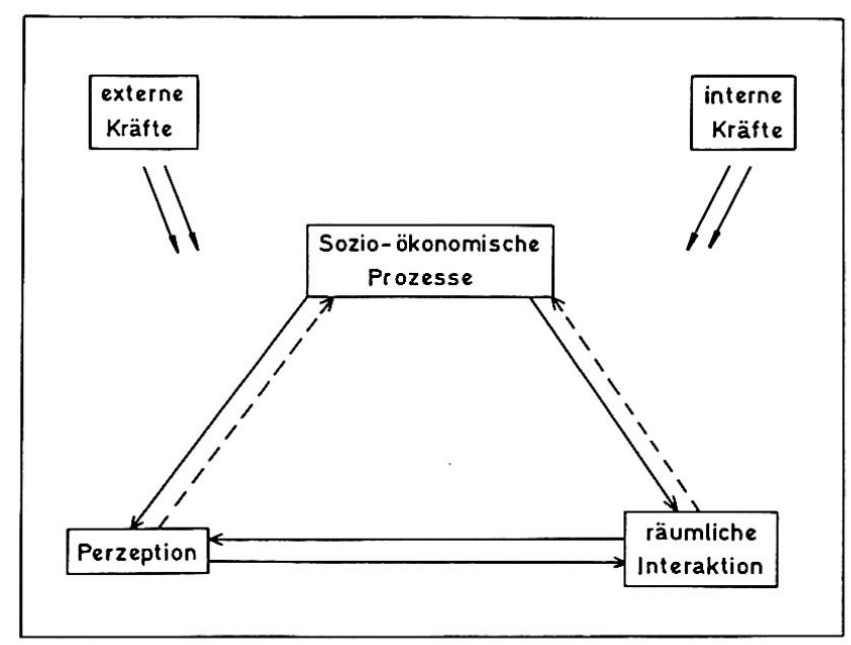

Fig.3: Das Zusammenspiel von Prozessen, Perzeption und Interaktion. 
ständigen Veränderung der Raumvorstellung (mental map) Anlass. Politische und sozio-ökonomische Prozesse steuern die Interaktion (über Standortentscheide, Lohn- und Preisentwicklung, Wechselkurse usw.) und wirken so wieder auf die mental map ein. Diese kann ihrerseits auf Standortentscheide einen gewissen Einfluss ausüben. ${ }^{3)}$ Im Falle der Grenzregion kommt als zusätzliche Komplikation hinzu, dass mehrere Prozessräume zu beachten sind. Während über die Bewegung über die Grenze hinweg gewisse Aussagen relativ leicht möglich sind (Leimgruber 1979 a), existiert kaum Material, das die Grenze in ihrem Einfluss auf den Wahrnehmungsraum untersucht. Auf diesen Ansatz haben Reynolds \& McNulty schon 1968 hingewiesen. Doch soll zusätzlich die Beziehung zwischen Wahrnehmungs- und Aktionsraum hergestellt und die Rolle der verschiedenen Prozessräume untersucht werden.

3) Es wäre eine interessante Aufgabe, der Frage nachzugehen, wie weit die Raumvorstellung entscheidender Persönlichkeiten in der Industrie

\section{Literaturverzeichnis}

BERRY, Brian J.L.: A paradigm for modern geography. In: Chorley, R.J. (Ed.): Directions in Geography. London 1973, p.3-21.

BOUSTEDT, 0.: Die Verhaltensweise der Bevölkerung als ein Faktor der Standortbestimmung. In: Beiträge zur Raunforschung. (FS H. Bobek), Wien 1964, p.9-20.

BROODFIELD, H.C.: On the environment as perceived. In: Progress in Geography 1, p.51-80.

BRUSA, Carlo: Evoluzione di un'immagine geografica: Il varesotto turistico. Torino 1979.

CANTER, David: The Psychology of Place. London 1977.

CATLING, S.: Maps and Cognitive maps: the young child's perception. Vortrag an der Jahresversammlung 1979 der Geographical Association, 18.4.79, London 1979.

CORNA PELLEGRINI, Giacomo: Geografia e percezione del1'ambiente. Atti del Colloquio internazionale de1 26.2.79, Milano 1979.

COX, Kevin R.: Man, location, and behavior. An introduction to human geography. New York 1972 .

COX, Kevin R. \& GOLLEDGE, R.G.: Behavioral problems in geography: a symposium. North western University, Dept. of Geography, studies in Geography, vol. 17, 1969.

DOHERTY, J.: Developments in Behavioural Geography. Graduate School of Geography, Discussion Paper, Nr. 35. London School of Economics, 1969.

DOWNS, Roger: Geographic space perception: past approaches and future prospects. In: Progress in Geography, vol.2, 1970, p.65-108.

GEIPEL, Robert: Probleme sozialräumlicher Jugendforschung. In: Der Erdkundeunterricht. Heft 19, Stuttgart 1974, p.4-10.

GOODEY, B.: Perception of the Environment. University of Birmingham, Centre for urban and regional studies, occasional paper $\mathrm{Nr} .17$, 1973.

\section{Schlussbemerkungen}

Die beiden letzten Beispiele zeigen, dass Perzeptionsstudien sehr wohl in einem weiteren humangeographischen Rahmen eingesetzt werden können. Gegenwärtig bewegt sich die Diskussion in der Perzeptionsforschung teilweise in methodischen Detailfragen, teilweise in der Erarbeitung von kognitiven Karten (mental maps, aber nicht im Sinne von Gould), und schliesslich entdeckt man Reiseführer und -berichte sowie Schriftste1ler als Schöpfer eines bestimmten räumlichen Vorstellungsbildes in der Vergangenheit. Alle diese Forschungsrichtungen haben im Sinne einer methodisch-theoretischen Weiterentwicklung eine bedeutende Aufgabe. Doch sollte die Perzeptionsforschung auch vermehrt auf die Anwendung ausgerichtet werden, damit der Mensch nicht nur in der geographischen Forschung, sondern auch in der planerischen Praxis vermehrt berücksichtigt wird.

die Standorte bedingt. Dieses Feld ist von der Perzeptionsforschung noch nicht betreten worden.

GOULD, Peter: On mental maps. Ann Arbor. In: R. DOWNS \& D. STEA (Ed.): Image and Environment. Chicago 1965/1973, p.182-220 (zitiert aus Nachdruck).

GOULD, Peter \& WHITE, Rodney: The mental maps of British school leavers. In: Regional studies, vol. 2, 1968, p.161-182.

GOULD, Peter \& WHITE, Rodney: Mental maps. Harmondsworth 1974.

HARTKE, Wolfgang: Gedanken über die Bestimmung vom Räumen gleichen sozialgeographischen Verhaltens. In: Erdkunde XIII, 1959, p.426-436 und: STORKEBADM, W. (Ed.): Sozialgeographie, Darmstadt 1969, p.162-186.

HAJOS, Anton: Wahrnehmungspsychologie. Stuttgart 1972 .

KISHIMOTO, Haruko: 'Mental maps" und Kartographie. In: Vermessung, Photogrammetrie, Kulturtechnik. Fachblatt 1-75, 1975, p.62-64.

LEIMGRUBER, Walter: I frontalieri nel Mendrisiotto 1978. Vervielfältigt. Basel 1979 a.

LEIMGRUBER, Walter: Percezione ambientale nella zona frontaliera del Canton Ticino. In: CORNA PELLEGRINI, G. (Ed.): Geografia e percezione dell'ambiente. Milano 1979 b.

LEY, David: The black inner city as a frontier outpost: images and behaviour of a North Philadelphia neighbourhood. Diss. (unveröff.), University Parle, Pennsylvania 1972.

LOWENTHAL, David (Ed.): Environmental Perception and Behaviour. Research Paper No.109, Dept. of Geography, Chicago 1967.

LYNCH, Kevin: The image of the city. Cambridge, Mass. 1960, 14. Auf1. 1977.

MERCER, Charles: Living in cities. Psychology and the urban environment. Harmondsworth 1975.

ORLEANS, Peter: Differential cognition of urban residents: effects of social scale on mapping. In: R. DOWNS \& D. STEA (Ed.): Image and Environment. Chicago 1973, p.115-130. 
REYNOLDS, David R. \& McNULTY, Michae1 L.: On the analysis of political boundaries as barriers: a perceptual approach. In: The East Lakes Geographer, vol. 4, 1968, p.21-38.

RUHL, Gernot: Das Image von München als 'Faktor für den Zuzug. Münchner Geographische Hefte Nr.35, 1971.

SAARINEN, Thomas F.: Perception of environment. Commission on College Geography Resource Paper No.5, 1969.

SAARINEN, Thomas F.: Student views of the world. In: R. DOWNS \& D. STEA (Ed.): Image and Environment. Chicago 1973, p.148-161.

SONNENFELD, Joseph: Geography, perception, and the behavioural environment. In: P.W. ENGLISH
\& R.C. MAYFIELD (Ed.): Man, space, and environment. New York 1972, p.244-251.

THOMALE, Eckhard: Geographische Verhaltensforschung. In: H. DICKEL et al.: Studenten in Marburg. Marburger Geographische Schriften, Heft 61, 1974, p.9-30.

TUAN, Yi-Fu: Topophilia. A study of environmental perception, attitudes, and values. Englewood C1iffs 1974

WOLPERT, Julian: Behavioural aspects of the decision to migrate. In: Papers, Regional Science Association, vol. 15, 1965. Zitiert aus Nachdruck in: P.W.ENGLISH \& R.C. MAYFIELD (Ed.) : Man, space, and environment. New York 1972, p.401-410.

\section{Literaturbesprechungen}

LICHTENBERGER, Elisabeth: Die Wiener Altstadt. Von der mittelalterlichen Bürgerstadt zur City. 410 S., 67 Fig., 4 Bildtafeln und 1 Kartenband mit 21 Karten, Verlag Franz Deuticke, Wien 1977, DM 140,-

Das vorliegende Werk vermittelt einen umfangreichen Ueberblick über die Entwicklung Wiens von der mittelalterlichen Bürgerstadt über die Viertelsbildung und soziale Gliederung um die Mitte des 16. Jahrhunderts und die Umwandlung der mittelalterlichen Bürgerstadt zur barocken Residenz. Weiterhin werden die Uebergangsperiode von der Residenz zur City, die Gründerzeit und die Umschichtungen seit der Zwischenkriegszeit erfasst. In der Betrachtensweise dominiert die Herausarbeitung der physiognomischen bautypologischen Substanz im Verein mit ihrer sozialgeographischen Determinierung. Verbunden mit dieser analytischen Arbeit ist der sozialökologische Ansatz, bei dem das Schwergewicht auf der Feststellung und Erklärung der Assoziation und Sukzession bestimmter Sozialgruppen und Wirtschaftsnutzungen liegt. Die Auswertung des ersten Hofquartierbuches aus dem Jahre 1563 bildet eine wesentliche Forschungsgrundlage. Im Verein mit der zweiten Aufnahme 1566 war es möglich, die bauliche Gestalt der Stadt und Stockwerkhöhe zu erfassen. Mit Hilfe der Angaben über Grösse und Zah1 der Wohnungen konnte das bürgerliche Miethauswesen aufgezeigt werden. Der Grosse Wert des Hofquartierbuches 1563 besteht darin, dass es die Berufe der Mieter angibt, womit ein fast vollständiges Bild der bürgerlichen Bevölkerung des frühneuzeitlichen Wiens gewonnen werden konnte. Es zeigt sich so, dass bereits im 16. Jahrhundert über $70 \%$ der Mieter in Kleinst- und Kleinwohnungen lebten. Es waren in erster Linie die grossen Wohnhöfe der Patriziergeschlechter, die sich in Miethäuser umgewandelt hatten, während die räumlich beengten Handwerkerhäuser nur beschränkte Möglichkeiten für die Aufnahme von Mietparteien boten. Ausserdem war um die Mitte des 16. Jahrhunderts die Zahl der behausten Handwerker bereits stark zusammengeschrumpft. In oft modellartiger Weise erhielten sich verschiedene mittelalterliche Standortgemeinschaften von Berufsgruppen bis herauf zur Neuzeit. Liegt so in der Darstellung Wiens um die Mitte des 16. Jahrhunderts ein hoher Originalitätsgrad begründet, so hängen die am Ende des Werkes herausgearbeiteten Prozesse, die in der Altstadt durch das Wachsen der städtischen Agglomeration ausgelöst wurde, sehr mit aktuellen Fragen der Raumordnung zusammen. Die Zeit der Barockresidenz steht unter dem Vorzeichen des Abschiebens der Gewerbetreibenden, deren Hilfskräfte und der Taglöhner aus der Altstadt in die Vorstädte; in der Manufakturzeit folgten die kleineren und mittleren Beamten und Angestellten. Im Zuge der hochund spätgründerzeitlichen Bevölkerungsabnahme stellten der Finanzadel, die bürgerliche Oberschicht und die freien Berufe aus der Altstadt weichende Sozialgruppen dar. Bis zum Ende des 18. Jahrhunderts blicb der an Läden gebundene Einzelhandel hauptsächlich auf die Altstadt beschränkt. Erst in der Gründerzeit kam es zu einer Dezentralisierung von Geschäftsarten aus der Altstadt. Die Gegenwart hat an den gründerzeitlichen Citystrassenstrukturen der Altstadt wenig geändert. Der Schrumpfungsprozess der City (Zusammenbruch der Monarchie) hat die meisten Viertelsbildungen der Wirtschaftscity aufgelöst. Bankenviertel, Zeitungsviertel und Viertel der Auskunfteien sind Beispiele für diesen Auflösungsprozess. Der gesamtheitliche altstädtische Extensivierungsprozess stellt ein schwerwiegendes Problem der heutigen Raumplanung dar.
GH $4 / 79$
Helmut Riedl, Salzburg 